\title{
Energy-loss straggling of swift heavy ions in an electron gas
}

\author{
I. Nagy, ${ }^{1,2}$ R. Vincent, ${ }^{2}$ J. I. Juaristi, ${ }^{3,4}$ and P. M. Echenique ${ }^{2,3,4}$ \\ ${ }^{1}$ Department of Theoretical Physics, Institute of Physics, Technical University of Budapest, H-1521 Budapest, Hungary \\ ${ }^{2}$ Donostia International Physics Center DIPC, P. Manuel de Lardizabal 4, 20018 San Sebastián, Spain \\ ${ }^{3}$ Departamento de Física de Materiales, Facultad de Químicas UPV/EHU, Apartado 1072, 20080 San Sebastián, Spain \\ ${ }^{4}$ Unidad de Física de Materiales, Centro Mixto CSIC-UPV/EHU, P. Manuel de Lardizabal 3, 20018 San Sebastián, Spain
}

(Received 4 June 2008; published 17 July 2008)

\begin{abstract}
Classical methods are employed to characterize the energy-loss straggling for swift, heavy ions interacting with independent electrons of a degenerate electron gas. The method of Bohr on the statistical aspects of the energy-loss process is used, and the electron-intruder collision is modeled via a finite-range screened Coulomb potential energy. The transport fluctuation cross section is calculated analytically within the framework of the impact-parameter method. Comparison with straggling data obtained for carbon foils with singly ionized carbon ions is made, and a good agreement is found in the validity range of the applied classical methods.
\end{abstract}

DOI: 10.1103/PhysRevA.78.012902

PACS number(s): $34.50 . \mathrm{Bw}$

\section{INTRODUCTION}

In the characterization of energy dissipation of heavy projectiles the average energy loss is a key quantity, both experimentally and theoretically. Sometimes, however, it is necessary to consider the fact that during penetration, the energy distribution of the beam is broadened. A monoenergetic beam is no longer monoenergetic after passing through a layer of matter. This happens because slowing of an external moving charge is the result of independent individual collision events, the number of which is governed by statistical laws.

By applying Bohr's theory [1] to the mean square fluctuation, $\Omega^{2}=\left\langle(\Delta E-\langle\Delta E\rangle)^{2}\right\rangle$ where $\Delta E$ is the energy loss, Lindhard and Sorensen [2] obtained for an electron gas

$$
\Omega^{2}=\left(\frac{T_{0}}{2}\right)^{2} \sigma_{t r f}(p)\left(n_{0} \Delta x\right),
$$

in which $\Delta x$ is the penetration length, $p=m v$ the momentum of electrons in relative motion, and $n_{0}$ the density of the electron gas. The maximal energy transfer is $T_{0}=m(2 v)^{2} / 2$ in a two-body collision between the swift heavy projectile moving with velocity $v$ and an electron. The transport fluctuation $(\operatorname{tr} f)$ cross section is defined as follows:

$$
\sigma_{t r f}(p)=\int d \sigma(1-\cos \theta)^{2},
$$

in terms of the differential cross section $(d \sigma)$ and the scattering angle $(\theta)$. Since $(1-\cos \theta)^{2}=2(1-\cos \theta)-\sin ^{2} \theta$, one easily gets the $\sigma_{t r f}(p)=2 \sigma_{t r}(p)-\sigma_{\eta}(p)$ representation in terms of transport $(t r)$ and viscosity $(\eta)$ cross sections [3]. Note that in Feynman's influence-functional theory [4] the transverse component of the force-force correlation function is proportional to the $\sigma_{\eta}(p)$ cross section for a fast heavy particle in an electron gas. The longitudinal component, similarly to straggling, is proportional to $\left[2 \sigma_{t r}(p)-\sigma_{\eta}(p)\right]$.

We stress that the applicability of Eq. (1) is subject to certain conditions in the case of a degenerate electron gas. In the theory of Bohr one accounts for straggling by dividing up collisions into a large number of statistically independent processes. Thus the mean number of collisions, into a given scattering angle, is simply $\left(n_{0} \Delta x\right) d \sigma(p, \theta)$. To apply Eq. (1) in our case, the ion velocity must be large compared to the Fermi velocity $v_{F}$. For rigorous investigations on the important role of the Pauli's constraint in allowed electronic final states at low $\left(v<v_{F}\right)$ ion velocities, we refer to earlier works $[5,6]$.

The unscreened, bare Coulombic case is well known from the pioneering work of Bohr on straggling, and serves as a natural limit to comparisons. This limit is independent of the applied scattering approaches in three dimensions, since for the bare interaction between charges the classical and quantum-mechanical methods give the same, charge-sign and Planck-constant independent, differential cross section. In reality, however, it is necessary to take into account the effect of screening, and investigate its influence in slowing down with a nonperturbative method. This goal is achieved in the present study via an analytical representation of straggling obtained with the classical potential-scattering method.

The rest of the paper is organized as follows. The next section, Sec. II, is devoted to the applied theoretical method and the obtained results. In Sec. III, a short summary is given.

\section{THEORY AND RESULTS}

In order to discuss the role of screening of a bare charge $(Z)$ in straggling, and thus characterize deviations from the unique Coulombic limit described by Bohr, we apply in the present study a well-known and simple potential energy to model the interaction,

$$
V(r)=-\frac{Z e^{2}}{r}\left(1-\frac{r}{R}\right),
$$

with the $V(r)=0$ constraint, for $r \geqslant R$. This form for a screened potential was used [3] in the partial-wave scattering description of electron-atom interactions at the beginning of applied quantum mechanics. It is the potential form by which [7] the effect of giant glory in classical atomic collisions was pointed out. The explanation of this effect is simple. 
The analytical expression which connects the observable scattering angle $(\theta)$ and the classical impact parameter $(b)$ is as follows [8] with the above potential:

$$
\tan ^{2}\left(\frac{\theta}{2}\right)=\left(\frac{Z e^{2}}{b m v^{2}}\right)^{2} \frac{1-(b / R)^{2}}{\left[1-\left(Z e^{2} / R\right) /\left(m v^{2}\right)\right]^{2}} .
$$

For $\left(Z e^{2} / R\right) /\left(m v^{2}\right)=1$, which can happen only for attractive $(Z>0)$ interactions, one gets $\theta \rightarrow \pi$ for all $0 \leqslant b \leqslant R$. Such a condition results in the giant-glory [7] effect. For $\left(Z e^{2} / R\right) /\left(m v^{2}\right)<1$, and with positive charges, only one trajectory contributes to the scattering at a given $\theta$. This is, of course, always the case for repulsive potentials $(Z<0)$. From now on, unless otherwise stated, we restrict ourselves to the range of $\left(Z e^{2} / R\right) /\left(m v^{2}\right) \leqslant 1$ and positive charges. We stress the point that the phenomenon of Coulomb glory, by which the domain of effective backscattering is extended in the impact-parameter scale, always exists [7] for screened attractive potentials. The phenomenon is particularly robust (giant) in the above finite-range potential.

The classical transport cross section has been derived earlier [8-10] to the characterization of the charge-sign $(Z>0$ and $Z<0$ ) effect in the energy loss. It is given by

$$
\sigma_{t r}^{c l}(v)=\pi R^{2} \frac{2}{(A-1)^{2}}[A \ln A-(A-1)],
$$

where $A=\left[m v^{2}\left(R / Z e^{2}\right)-1\right]^{2}$ is shorthand. The maximum value in the $\sigma_{t r}^{c l} /\left(\pi R^{2}\right)$ dimensionless quantity appears at $\left(Z e^{2} / R\right) /\left(m v^{2}\right)=1$. The applicability range of the above cross section has been investigated [11] in detail, via a comparison with results based on numerically exact quantum calculations, and the condition of $\left(Z e^{2} / R\right) /\left(m v^{2}\right)<1$ has been deduced for positive charges. For $\left(Z e^{2} / R\right) /\left(m v^{2}\right)>1$, the quantum-mechanical calculation gives oscillations (at given $R$ and $v$ ) in the transport cross section as a function of $Z$.

Based on the equation for the scattering angle, we obtain for the classical viscosity cross section the following expression:

$$
\sigma_{\eta}^{c l}(v)=\pi R^{2} \frac{4}{(A-1)^{2}}\left[\left(\frac{A+1}{A-1}\right) A \ln A-2 A\right] .
$$

This cross section is zero at $\left(Z e^{2} / R\right) /\left(m v^{2}\right)=1$, and at $\left(Z e^{2} / R\right) /\left(m v^{2}\right)=1 / 2$ appears its maximum. The transport fluctuation cross section becomes

$$
\sigma_{t r f}^{c l}(v)=\pi R^{2} \frac{4}{(A-1)^{2}}\left[(A+1)-\left(\frac{2 A}{A-1}\right) \ln A\right] .
$$

It is easy to see that in the $\left|\left(Z e^{2} / R\right) /\left(m v^{2}\right)\right| \rightarrow 0$ limit one gets to leading order

$$
\begin{aligned}
& \sigma_{t r}^{c l}(v) \simeq \frac{4 \pi Z^{2} e^{4}}{m^{2} v^{4}}\left(1+2 \frac{Z e^{2}}{R m v^{2}}\right)\left(\ln \frac{R m v^{2}}{\left|Z e^{2}\right|}-\frac{1}{2}\right), \\
& \sigma_{\eta}^{c l}(v) \simeq \frac{8 \pi Z^{2} e^{4}}{m^{2} v^{4}}\left(1+2 \frac{Z e^{2}}{R m v^{2}}\right)\left(\ln \frac{R m v^{2}}{\left|Z e^{2}\right|}-1\right) .
\end{aligned}
$$

These separate cross sections are logarithmically divergent in the bare Coulomb $(R \rightarrow \infty)$ limit. For finite $R$ values they encode a charge-sign [12] effect. Their properly weighted sum is not divergent in the above asymptotic limit

$$
\sigma_{t r f}^{c l}(v) \simeq \frac{4 \pi Z^{2} e^{4}}{m^{2} v^{4}}\left(1+2 \frac{Z e^{2}}{R m v^{2}}\right),
$$

and yields the Coulombic Bohr's $\sigma_{t r f}^{C}(v)=4 \pi Z^{2} e^{4} /\left(m v^{2}\right)^{2}$ to the straggling when the charge-sign-dependent term in the parentheses becomes negligible.

Now, we define the $\left[\Omega^{2} / \Omega_{B}^{2}\right]=\left[\sigma_{\operatorname{trf} f}^{c l} \sigma_{t r f}^{C}\right]$ classical ratio, and investigate its behaviors for attractive $(Z>0)$ potentials. In terms of the $A$ variable we get for this ratio

$$
\frac{\Omega^{2}}{\Omega_{B}^{2}}=\frac{1}{(1-\sqrt{A})^{2}}\left[(A+1)-\left(\frac{2 A}{A-1}\right) \ln A\right],
$$

For $\left[\left(Z e^{2} / R\right) /\left(m v^{2}\right)\right] \rightarrow 0$ this traditional ratio tends to unity. At the $\left[\left(Z e^{2} / R\right) /\left(m v^{2}\right)\right]=1$ value we get $\left[\Omega^{2} / \Omega_{B}^{2}\right]=1$ also. Between these limits, at $\left[\left(Z e^{2} / R\right) /\left(m v^{2}\right)\right]=1 / 2$, the ratio is maximal with $\left[\Omega^{2} / \Omega_{B}^{2}\right]=4 / 3$. Thus, we obtain the biggest value when there is a maximum in the viscosity cross section also. The velocity range which corresponds to $\left[\left(Z e^{2} / R\right) /\left(m v^{2}\right)\right] \in[0,0.5]$ at fixed $R$ and $Z$, heralds a plateaulike character in straggling as a function of the intruder velocity. At $\left[\left(Z e^{2} / R\right) /\left(m v^{2}\right)\right]>1$ the ratio is given by

$$
\frac{\Omega^{2}}{\Omega_{B}^{2}}=\frac{1}{(1+\sqrt{A})^{2}}\left[(A+1)-\left(\frac{2 A}{A-1}\right) \ln A\right] .
$$

We shall return to this form below, when we discuss the strong-field effect at fixed $R$ and $v$, by varying the positive charge $Z$ of intruders.

The quantum-mechanical [3] expressions for the cross sections introduced above are

$$
\begin{gathered}
\sigma_{t r}(k)=\frac{4 \pi}{k^{2}} \sum_{l=0}^{\infty}(l+1) \sin ^{2}\left[\delta_{l}(k)-\delta_{l+1}(k)\right], \\
\sigma_{\eta}(k)=\frac{4 \pi}{k^{2}} \sum_{l=0}^{\infty} \frac{(l+1)(l+2)}{2 l+3} \sin ^{2}\left[\delta_{l}(k)-\delta_{l+2}(k)\right],
\end{gathered}
$$

in which $\delta_{l}(k)$ are the phase shifts and $k=m v / \hbar$. The quantum-mechanical evaluation [2] with bare Coulomb interaction energy is based on repeated use of the

$$
\delta_{l}(k)-\delta_{l+1}(k)=\arctan \left[\frac{\kappa / 2}{(l+1)}\right] \text {. }
$$

exact expression, and results in the form of $\sigma_{\operatorname{trf}}^{C}(k)$ $=\left(\pi / k^{2}\right) \kappa^{2}$ in terms of the dimensionless Bohr parameter $\kappa$ $=2 Z e^{2} /(v \hbar)$ and $k$. This form for $\sigma_{\operatorname{tr} f}$ does not depend on $\hbar$. Of course, without screening the cross sections are, separately, logarithmically divergent, as in the classical treatment above. But the $\left(2 \sigma_{t r}-\sigma_{\eta}\right)$ weighted sum of them is finite, and gives the $\hbar$-independent classical expression of Bohr.

Lindhard and Sorensen made a careful investigation of a Bloch-like $\left(\propto Z^{4}\right)$ correction in straggling using the classical interpretation of the angular momentum [2]. Of course, with a bare Coulomb interaction energy there is no such term. On the other hand, the effect of screening modifies this statement. The higher-order expansion of the classical Eq. (7) for the $\left[\left(Z e^{2} / R\right) /\left(m v^{2}\right)\right] \rightarrow 0$ perturbative limit gives 


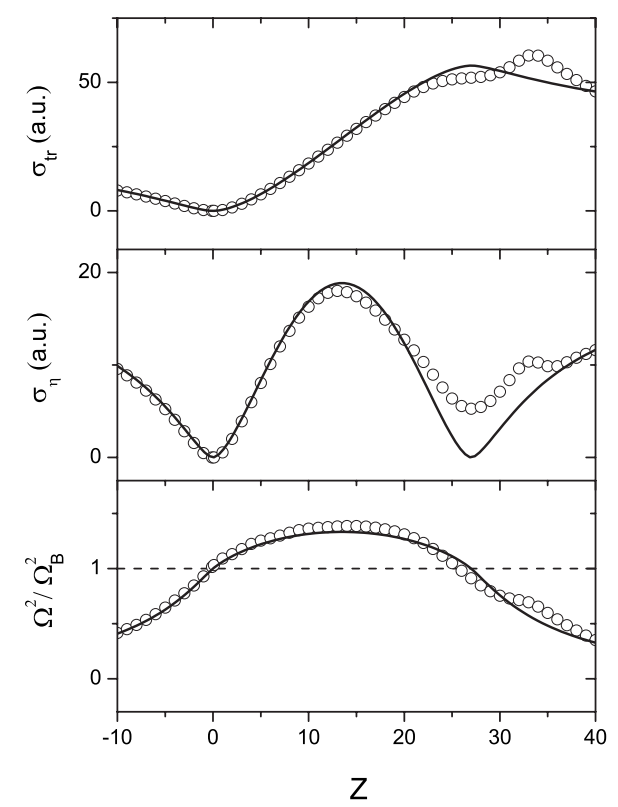

FIG. 1. Cross sections and straggling ratios, as a function of $Z$. The screening parameter is $R=3$, and the velocity is $v=k=3$, both in atomic units (a.u.). Solid curves are based on the classical method, while the open circles refer to the wave-mechanical calculations.

$$
\sigma_{t r f}^{c l}(v) \simeq \frac{4 \pi Z^{2} e^{4}}{m^{2} v^{4}}\left(1+2 \frac{Z e^{2}}{R m v^{2}}\right)\left[1-\left(\frac{2 Z e^{2}}{R m v^{2}}\right)^{2} \ln \frac{R m v^{2}}{\left|Z e^{2}\right|}\right]
$$

This shows that an earlier statement [13] on the absence of a $Z^{4}$-proportional term in energy-loss straggling is valid only for unscreened potentials.

Now we discuss how the Coulomb limit arises from the first-order Born $(B)$ approximation in quantum mechanics with our screened potential. This method results in

$$
\sigma_{t r f}^{(B)}(k)=\frac{4 \pi Z^{2} e^{4}}{m^{2} v^{4}}\left(1-\frac{1}{2(k R)^{2}} \int_{0}^{2 k R} d x x j_{0}(x)\left[1-j_{0}(x)\right]\right),
$$

where $j_{0}(x)=\sin x / x$. The prefactor of the integral is proportional to $\hbar^{2} /(m v R)^{2}$. Due to the highly oscillating character of the above integrand in an $\hbar \rightarrow 0$ limit, the problem is somewhat similar to the corresponding limit in the Mott cross section for scattering of identical fermions. As is well known, there a purely quantum-mechanical interference term disappears in the classical limit [3].

After the above details on cross sections in classical and quantum mechanics, we turn our attention to the quantitative results obtained in this study. Illustrative results for the cross sections and straggling ratios are exhibited in Fig. 1, at fixed $v=3$ (a.u.) and $R=3$ (a.u.), as a function of the charge $Z$. This parameter set represents $[11,14]$ the important case of highly charged fast ions. Note that for $Z>27$ we are out of the $\left(Z e^{2} / R\right) /\left(m v^{2}\right)<1$ range, and the expression given by Eq. (12) holds for the straggling ratio. But this is precisely the range $(Z>27)$ where quantum oscillations set in, in both

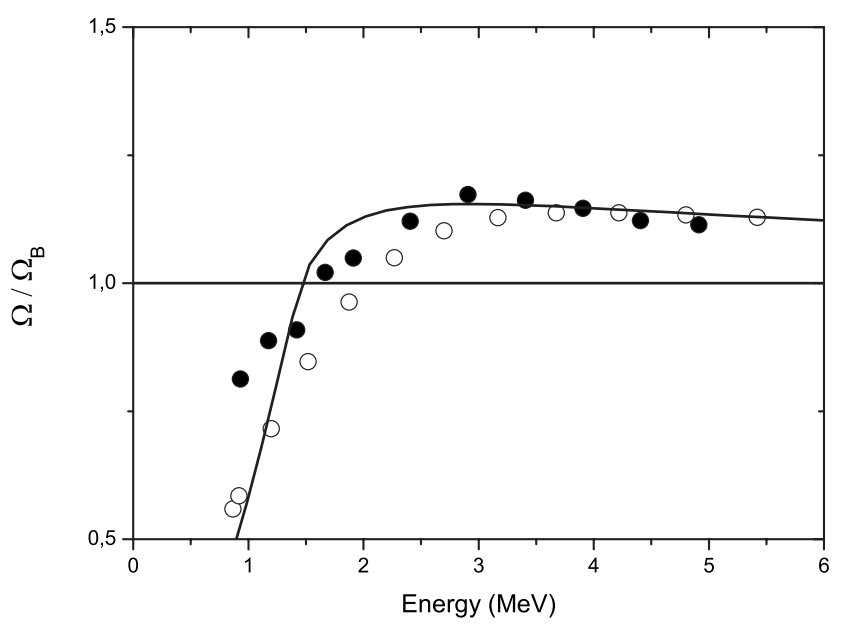

FIG. 2. Straggling ratios, $\Omega / \Omega_{B}$, as a function of the ion energy. The screening parameter is $R=1.23$ in atomic units. The experimental points are denoted by solid circles, the solid curve is based on the classical approximation, and open circles refer to the wavemechanical results.

cross sections. The straggling ratios show, on the other hand, a quite smooth character, due to certain cancellations in the weighted sum $\left(2 \sigma_{t r}-\sigma_{\eta}\right)$. Similar smooth characters were obtained (not shown) with other fixed parameters for $R$ and $v$, as a function of $Z$, for $\left(Z e^{2} / R\right) /\left(m v^{2}\right)>1$. Therefore, it seems that the $(1-\cos \theta)^{2}$ factor in Eq. (2) acts to eliminate, to a great extent, those effects which are characteristics of the wave-mechanical description.

The values of input variables $\{Z, R, v\}$ depend, of course, on concrete physics in both the classical and quantummechanical descriptions. Figure 2 shows a comparison of our theoretical results for straggling with recent data obtained [15] in carbon foils by carbon ions $(Z=6)$. The screening parameter, $R=1.23$ in a.u., is taken from Slater's table [16]. The data for the energy-loss straggling are denoted by solid circles. The conversion between ion energy $(E)$ and ion velocity $(v)$ is $E(\mathrm{MeV})=0.3[v(\text { a.u. })]^{2}$ for carbon ions. There is a very good agreement at higher velocities, i.e., where the ion velocity is higher than the Fermi velocity, $v_{F} \approx 1.2$ in atomic units (a.u.). Practically, for about $v>2 v_{F}$, we can use the so-called "electron gas at rest" picture in order to apply Bohr's classical treatment on scattering events needed in his derivation of the energy-loss straggling.

At lower velocities one can see a growing deviation of the theoretical results from data, obtained by the transmission technique in thick $\left(15 \mu \mathrm{g} / \mathrm{cm}^{2}\right)$ carbon foil by carbon ions. The observed deviation suggests that within the framework of an electron gas model for a real solid target, a precise treatment on the quantum-statistical aspects of allowed electronic excitations from the Fermi sea is needed when the ion velocity is comparable with the Fermi velocity, where the role of charge exchange can $[17,18]$ also be important. Separate consideration of different charge-state channels may need, in principle, different interaction potentials, i.e., certain modifications of the form given by Eq. (3). On the other hand, Eq. (3) represents [3,16] an atomic screening and thus provides a realistic modeling of the most important [cf. Eq. (2)] short-range part of interaction energies for singly ionized intruders. 
Furthermore, although in the transmission technique of data-derivation scatterings events with small impact parameters (large-angle scattering) between the intruder and a target atom are of minor importance, the overlap of atomic electron clouds during transmission can result in effects not considered in a homogeneous electron gas model for solid targets. In this respect, the information obtained by the atomic analog of energy-loss straggling could help to establish a more detailed explanation of the observed deviation. The nonperturbative quantum treatment by Grande and Schiwietz [19] on energy-loss straggling of bare incident ions on $\mathrm{H}$ and $\mathrm{He}$ atoms indicates that the effects of charge-state variations, ionization of a localized bound electron, and dielectronic excitations (electron-electron collisions) are important ingredients of a quantitative description. During transmission, such dielectronic excitations in the overlapping electron clouds can enhance the energy-loss straggling at lower energies, and thus contribute to our understanding of deviations from data.

\section{CONCLUSIONS}

Motivated by the experimental and theoretical importance of swift, heavy ion energy loss straggling in condensed matter, the classical potential-scattering approach is investigated for an electron gas target. A physically motivated finite-range potential is applied to the present study, in which the numerically exact wave-mechanical results serve as a background. It is found that the classical description can provide quantitatively accurate results in the screened-potential case under the $\left[\left(Z e^{2} / R\right) /\left(m v^{2}\right)\right]<1$ condition for positive charges.

In the present model for the electron-intruder interaction energy, the $\Omega / \Omega_{B}$ ratio has a maximum at $\left[\left(Z e^{2} / R\right) /\left(m v^{2}\right)\right]$
$=1 / 2$. This is the value at which the maximum appears in the $\sigma_{\eta}^{c l}$ viscosity cross section also. Quite remarkably, our maximum value in $\Omega^{2} / \Omega_{B}^{2}$ seems to be very close to the one calculated numerically [13] in the classical binary theory with the atomic model for a target material. This suggests a universal-like behavior in $\Omega / \Omega_{B}$.

The agreement with data, obtained for carbon foils with carbon ions in the $E \in[1,5] \mathrm{MeV}$ energy range, is excellent in the high-velocity range where the kinematical conditions behind Bohr's prescription are satisfied. This agreement suggests to us that in the concrete case $(Z=6)$ investigated here via an electron gas model and effective interaction energy, the input parameters $\left(R\right.$ and $\left.v_{F}\right)$ are quite realistic to characterize the straggling.

The smooth character found in our potential-scattering study for the theoretical straggling ratio as a function of $Z$ even for strong fields $\left[\left(Z e^{2} / R\right) /\left(m v^{2}\right)>1\right.$, i.e., $(\kappa / 2) /(k R)$ $>1]$, can give a challenge to future studies within nonperturbative methods, in order to establish the precise limitations of the classical method applied to various moments of the loss process. Particularly, application of advanced semiclassical methods [20,21] can give further physical insight into the smooth character found here in an integrated cross section.

\section{ACKNOWLEDGMENTS}

We thank Professor J. Y. Hsu for valuable information on straggling experiments. One of us (I.N.) has been partially supported by the Hungarian OTKA (Grant No. T049571). R.V., J.I.J., and P.M.E. acknowledge support by the Basque Departamento de Educación, and the Spanish MCyT (Grant No. FIS2007-066711-CO2-00).
[1] N. Bohr, Mat. Fys. Medd. K. Dan. Vidensk. Selsk. 18, 1 (1948).

[2] J. Lindhard and A. H. Sorensen, Phys. Rev. A 53, 2443 (1996).

[3] N. F. Mott and H. S. W. Massey, The Theory of Atomic Collision (Clarendon Press, Oxford, 1965).

[4] L. Bönig, K. Schönhammer, and W. Zwerger, Phys. Rev. B 46, 855 (1992).

[5] P. Sigmund, Phys. Rev. A 26, 2497 (1982).

[6] P. M. Echenique, I. Nagy, and A. Arnau, Int. J. Quantum Chem. 23, 521 (1989).

[7] Yu. N. Demkov and V. N. Ostrovsky, J. Phys. B 34, L595 (2001), and references therein.

[8] I. Nagy, Nucl. Instrum. Methods Phys. Res. B 94, 377 (1994).

[9] I. Nagy and B. Apagyi, Adv. Quantum Chem. 46, 268 (2004).

[10] N. R. Arista, P. L. Grande, and A. F. Lifschitz, Phys. Rev. A 70, 042902 (2004).

[11] R. Vincent, J. I. Juaristi, and I. Nagy, Phys. Rev. A 71, 062902
(2005)

[12] W. H. Barkas, N. J. Dyer, and H. H. Heckman, Phys. Rev. Lett. 11, 26 (1963).

[13] P. Sigmund and A. Schinner, Eur. Phys. J. D 23, 201 (2003).

[14] J. I. Juaristi, A. Arnau, P. M. Echenique, C. Auth, and H. Winter, Phys. Rev. Lett. 82, 1048 (1999).

[15] J. Y. Hsu, Y. C. Yu, K. M. Chen, and C. H. Wang, Nucl. Instrum. Methods Phys. Res. B 266, 1170 (2008).

[16] J. C. Slater, Phys. Rev. 36, 57 (1930).

[17] A. Narmann, R. Monreal, P. M. Echenique, F. Flores, W. Heiland, and S. Schubert, Phys. Rev. Lett. 64, 1601 (1990).

[18] A. Narmann, W. Heiland, R. Monreal, F. Flores, and P. M. Echenique, Phys. Rev. B 44, 2003 (1991).

[19] P. L. Grande and G. Schiwietz, Phys. Rev. A 44, 2984 (1991).

[20] W. F. Egelhoff, Phys. Rev. Lett. 71, 2883 (1993).

[21] H. Friedrich, Theoretical Atomic Physics, 2nd ed. (SpringerVerlag, Berlin, 1998). 